\title{
Evaluation of the Insall-Salvati Ratio Among the Vietnamese Population: Application for Diagnosis of Patellar Malalignment
}

\author{
Thu Le Hoang $\mathrm{Di}^{1}$ \\ Thanh Hoang Ngoc (D) \\ Dac Hong An Ngo (i) \\ Nghi Thanh Nhan Le ${ }^{2}$ \\ Binh Le Trong (D)' \\ Khoan Le Trong' \\ Thao Nguyen Thanh (D) \\ 'Department of Radiology, Hue \\ University of Medicine and Pharmacy, \\ Hue University, Hue, Vietnam; \\ ${ }^{2}$ Department of Surgery, Hue University \\ of Medicine and Pharmacy, Hue \\ University, Hue, Vietnam
}

\begin{abstract}
Introduction and Purpose: An abnormal patellar position has been proven to be associated with anterior knee pain and several other conditions that affect the patellofemoral joint. The purpose of this study was to determine the incidence of patella alta and patella baja and the applicability of the normal range of the Insall-Salvati ratio in the Vietnamese population.
\end{abstract}

Methodology: Magnetic resonance imaging (MRI) was used to examine 455 Vietnamese subjects' knees. The Insall-Salvati ratio - the ratio of patellar ligament length (LL) and patellar length (PL) — was measured using sagittal T1-weighted images.

Results: The overall mean LL/PL ratio was 1.02 (standard deviation 0.15 ). No significant differences in the LL/PL ratio were observed between sexes. The frequencies of patella alta (Insall-Salvati ratio $>1.32$ ) and patella baja (Insall-Salvati ratio $<0.72$ ) were $0.9 \%$ and $2.4 \%$, respectively.

Conclusion: The Insall-Salvati ratio is applicable to Vietnamese populations in which high flexion activities such as kneeling and sitting cross-legged are customary. According to our measurement, the normal range of the ratio among Vietnamese subjects was 0.72 to 1.32 .

Keywords: Insall-Salvati ratio, patellofemoral joint, anterior knee pain, MRI, Vietnamese

\section{Introduction}

Anterior knee pain is commonly found among young physically active adults and adolescents. $^{1-3}$ Its pathogenesis is multifactorial. ${ }^{1}$ Patellar malalignment has been recently suggested to be an important factor contributing to the mechanism of anterior knee pain. ${ }^{4}$ In addition to several other conditions that affect the patellofemoral joint, patella alta and patella baja are among the most common patellar pathologies encountered by physicians and radiologists. ${ }^{5}$ The Insall-Salvati ratio has been used to evaluate patellar height. ${ }^{6}$ This ratio is calculated by comparing the diagonal length of the patella with the patellar ligament. ${ }^{6}$ Insall and Salvati suggested a method to determine the ratio on radiographs. ${ }^{6}$ However, magnetic resonance imaging (MRI) has proven to be an effective and accurate tool to calculate the Insall-Salvati ratio. ${ }^{7,8}$ MRI allows the accurate measurement of the patellar length and the length of the patellar ligament without the risk of radiation exposure. ${ }^{8}$ The patellar morphology, however, varies according to genetics, lifestyle, and social and cultural practices. ${ }^{9}$ People with squatting or kneeling habits, such as those who live in Southeast Asia, or individuals who frequently practice yoga, such as residents of India and other Asian countries, may also have different morphologies than
Correspondence: Thao Nguyen Thanh Department of Radiology, Hue University of Medicine and Pharmacy, Hue University, Hue, 53000, Vietnam Tel +84906449964

Email ntthao@hueuni.edu.vn 
Western populations. ${ }^{9,10}$ Studies have shown great variety in patellar morphology among ethnic groups. ${ }^{10-12}$ Differences in the Insall-Salvati ratio between sexes have also been reported. ${ }^{9,13}$ Thus, the widely accepted values of the InsallSalvati ratio for Western populations may not be applicable in Asian populations. Ratios specific to different ethnic groups would be of great benefit for practicing clinicians and radiologists when evaluating patellar position via MRI or radiographs. Therefore, we conducted this study to explore the normal range of the Insall-Salvati ratio and to determine the incidence of patella alta and patella baja in the Vietnamese population.

\section{Methodology}

Subjects were recruited from a hospital in central Vietnam. Inclusion criteria was individuals aged $\geq 18$ years old. MRIs of 455 knees were taken to measure the length of the patella and the patellar ligament. The patients were referred for MRI for various reasons. For purposes of this study, exclusion criteria were a history of knee fracture, knee dislocation or surgery. All patients were scanned using a 1.5 Tesla MRI scanner (Siemens, Erlangen, Germany) from February 2020 to February 2021. Knee images were obtained using sagittal images without fat saturation (TE: $12.0 \mathrm{~ms}$; TR: $552 \mathrm{~ms}$; acquisition matrix: $320 \times 320$ ) with the knees in $0^{\circ}$ of flexion. Image processing and measurement were carried out on a workstation provided by the manufacturer. The patellar ligament length (LL) and the patellar length (PL) were measured using mid-sagittal T1-weighted images (Figure 1). The patellar length was measured from the superior articular margin to the distal anterior tip of the patella. The patellar ligament length was measured along the posterior margin of the ligament from the patellar attachment to the tibial insertion. The LL/PL ratio was calculated. All measurements were carried out by two radiologists, one with more than 15 years of experience and the other with 3 years of experience. If they disagreed on a measurement, they discussed the issue and reached a final conclusion by consensus. The mean LL/PL ratio and the standard deviation (SD) were calculated using IBM SPSS Statistics version 24. LL/PL ratios higher or lower than the 2SD range were considered abnormal. Student's $t$ test was used to test the difference between sexes, with $p<0.05$ considered statistically significant. This study was approved by the Ethics Committee of Hue University of Medicine and Pharmacy, Hue University, Vietnam. Patients gave informed consent.

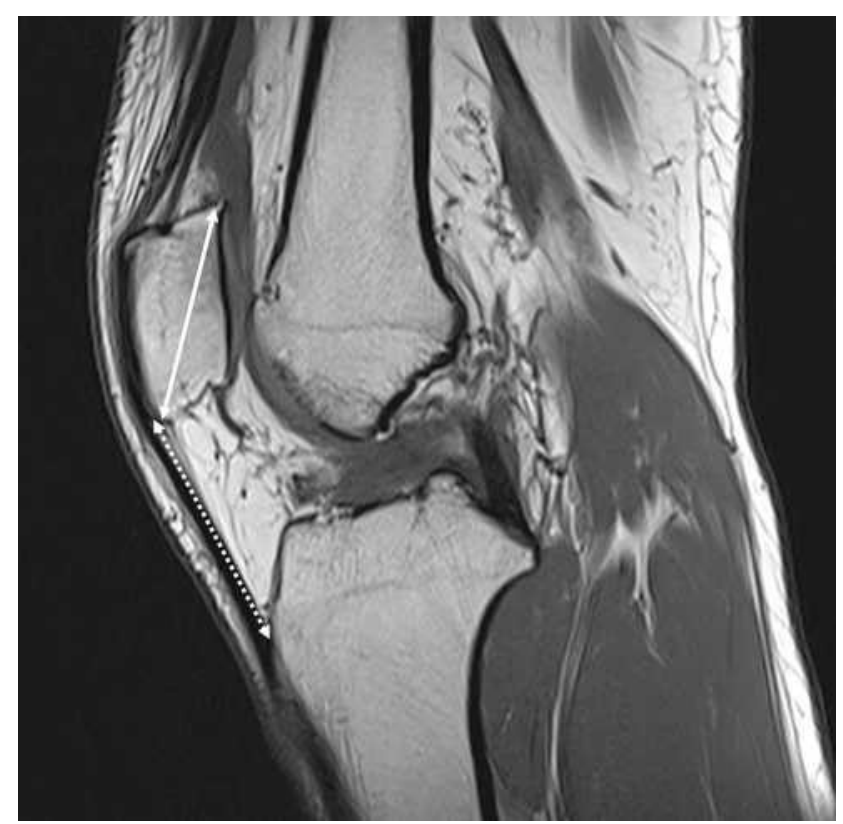

Figure I Measurement of patellar height and the length of the patellar ligament. The patellar length (white double arrow) was measured from the superior articular margin to the distal anterior tip of the patella. The patellar ligament length (white dashed double arrow) was measured along the posterior margin of the ligament from the patellar attachment to the tibial insertion.

\section{Results}

The data were in a left-skewed distribution (Figure 2). There was good intra- and inter-observer correlation. The overall mean LL/PL ratio was 1.02 (SD 0.15) for both sexes. No significant differences in the LL/PL ratio were observed between sexes (Table 1). Patella alta and patella baja were defined as LL/PL ratios greater than 1.32 and less than 0.72 , respectively. The frequencies of patella alta and patella baja were $0.9 \%$ and $2.4 \%$, respectively.

\section{Discussion}

The main finding of this study was that the normal range of the Insall-Salvati ratio among the Vietnamese population was 0.72 to 1.32 . Given that patellar malalignment may play an important role in anterior knee pain, accurate diagnosis of the patellar position is crucial for treatment and prognosis. Interestingly, our results overlap substantially with Western and Nigerian populations. ${ }^{6,8,14,15}$ However, the cutoff value for patella alta and patella baja in our study was lower than that in studies of Indian and Emirati populations ${ }^{10,11}$ but slightly higher than that in Korean populations (Table 2). ${ }^{9}$ Our findings, along with those of previous studies, suggest a great variety in the Insall-Salvati ratio across ethnicities. Sex had no effect on the Insall-Salvati ratio in the Vietnamese 


\section{Insall - Salvati ratio}

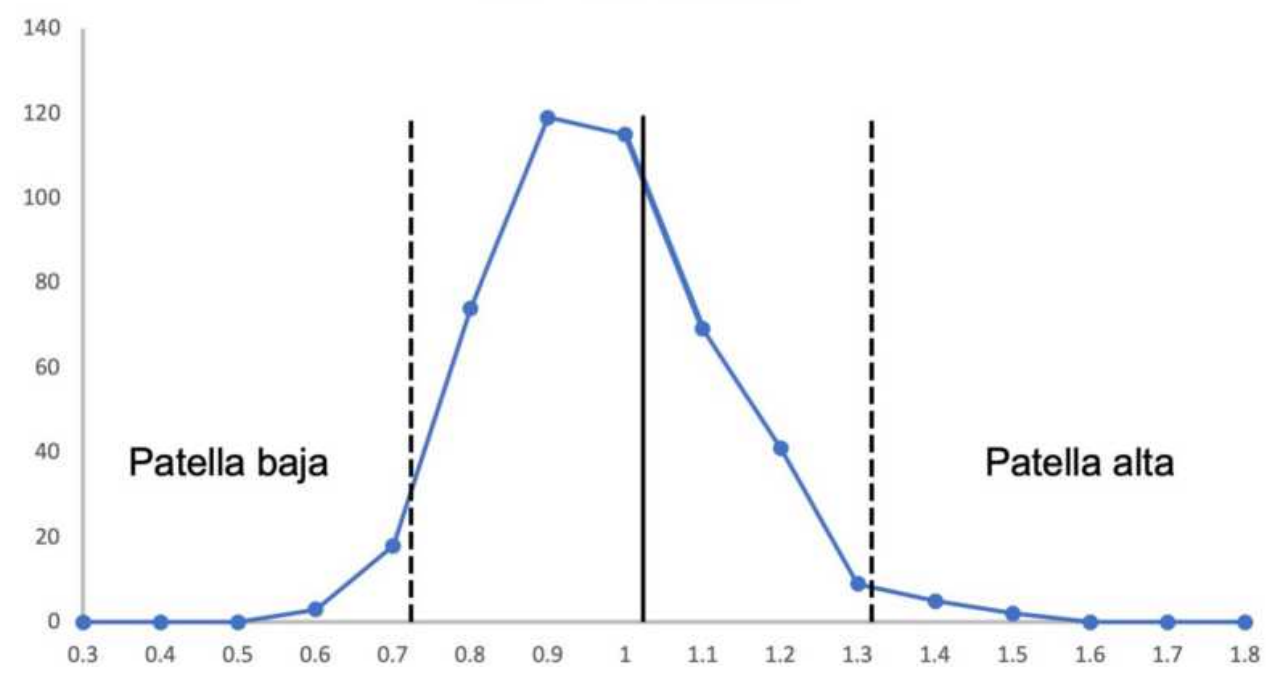

Figure 2 The distribution of the Insall-Salvati ratio for the whole sample. The thick solid line shows the mean (I.02), and the broken lines show the normal range within the 2 SD range $(0.72,1.32)$.

population. This finding is in line with those of some reported studies, ${ }^{8,16}$ although other studies have found sex differences. ${ }^{9,12,13}$ This inconsistent findings may be related to ethnic differences, sampling bias or differences in measurement methodology.

The frequencies of patella alta (Insall-Salvati ratio $>1.32$ ) and patella baja (Insall-Salvati ratio $<0.72$ ) were $0.9 \%$ and $2.4 \%$, respectively. Patellar alta appeared less common in the Vietnamese population than in the Korean group (0.9 vs $1.9 \%$ ), respectively, but patellar baja was more common in the Vietnamese population than in the Korean population (2.4 vs $2.11 \%$ ) using the same measurement method. ${ }^{9}$ In both populations, patella alta was less common than patella baja. In the Indian population, patella alta appeared more common than patellar baja $(2.8 \%$ and $1.0 \%$, respectively) (Table 3). ${ }^{10}$ These inconsistent findings again reflect the great diversity of patellar positions across ethnicities.

Patella malalignment may result in patellofemoral pain, instability, restricted motion and osteoarthritis. ${ }^{9,17,18}$ Surgical repair can restore knee functionality with overall favorable outcomes. ${ }^{19}$ However, one should keep in mind that anterior knee pain has multifactorial etiologies. The diagnosis of anterior knee pain remains controversial. ${ }^{3}$ A clear correlation is often lacking between structural abnormalities and clinical symptoms. ${ }^{3}$ Clinical diagnosis and imaging techniques are usually indicated to evaluate associated structural changes, if

Table I Insall-Salvati Ratio According to Age and Sex

\begin{tabular}{|c|c|c|c|c|}
\hline & $\begin{array}{c}\text { Total Patients } \\
(n=455)\end{array}$ & $\begin{array}{l}\text { Female } \\
(n=208)\end{array}$ & $\begin{array}{c}\text { Male } \\
(n=247)\end{array}$ & $\begin{array}{c}\text { p-value } \\
\text { (Female versus Male) }\end{array}$ \\
\hline & $\begin{array}{c}\text { Mean } \pm \text { SD } \\
\text { (Range) }\end{array}$ & $\begin{array}{c}\text { Mean } \pm \text { SD } \\
\text { (Range) }\end{array}$ & $\begin{array}{c}\text { Mean } \pm \text { SD } \\
\text { (Range) }\end{array}$ & \\
\hline Age & $\begin{array}{c}40.19 \pm 14.63 \\
(18,95)\end{array}$ & $\begin{array}{c}46.58 \pm 14.62 \\
(18,95)\end{array}$ & $\begin{array}{c}34.82 \pm 12.3 \\
(18,79)\end{array}$ & $<0.05$ \\
\hline LL $(\mathrm{cm})$ & $\begin{array}{l}4.06 \pm 0.52 \\
(2.59,5.65)\end{array}$ & $\begin{array}{l}3.88 \pm 0.47 \\
(2.59,5.09)\end{array}$ & $\begin{array}{l}4.21 \pm 0.52 \\
(2.75,5.65)\end{array}$ & $<0.05$ \\
\hline PL (cm) & $\begin{array}{c}4 \pm 0.36 \\
(3.11,5.17)\end{array}$ & $\begin{array}{c}3.76 \pm 0.28 \\
(3.11,4.9)\end{array}$ & $\begin{array}{l}4.19 \pm 0.3 \\
(3.2,5.17)\end{array}$ & $<0.05$ \\
\hline LL/PL ratio & $\begin{array}{l}1.02 \pm 0.15 \\
(0.66,1.59)\end{array}$ & $\begin{array}{l}1.03 \pm 0.15 \\
(0.76,1.59)\end{array}$ & $\begin{array}{l}1.01 \pm 0.15 \\
(0.66,1.48)\end{array}$ & 0.064 \\
\hline
\end{tabular}

Abbreviations: LL, ligament length; PL, patellar length; SD, standard deviation. 
Table 2 Mean Insall-Salvati Ratio and Standard Deviation of Different Studies

\begin{tabular}{|l|l|}
\hline Study & Mean Insall-Salvati Ratio \pm SD \\
\hline Our study & $1.02 \pm 0.15$ \\
Hong et al $^{9}$ & $0.92 \pm 0.14$ \\
Insall and Salvati $^{6}$ & $1.02 \pm 0.13$ \\
Althani et al $^{11}$ & $1.20 \pm 0.17$ \\
Schlenzka and Schwesinger $^{14}$ & $1.00 \pm 0.14$ \\
Ahmed et al $^{15}$ & $1.02 \pm 0.13$ \\
Miller et al $^{8}$ & $1.1 \pm 0.1$ \\
Upadhyay et al $^{10}$ & $1.14 \pm 0.18$ \\
\hline
\end{tabular}

Abbreviation: SD, standard deviation.

Table 3 The Prevalence of Patella Alta and Patella Baja in Different Populations

\begin{tabular}{|l|l|l|}
\hline Study & Patella Alta & Patella Baja \\
\hline Our study & $0.9 \%$ & $2.4 \%$ \\
Hong et al $^{9}$ & $1.9 \%$ & $2.11 \%$ \\
Upadhyay et al $^{10}$ & $2.8 \%$ & $1.0 \%$ \\
\hline
\end{tabular}

any. ${ }^{20}$ Avoiding unnecessary surgery is as important as identifying the etiology. Therefore, one should be very cautious when establishing a diagnosis of patellofemoral joint disorder. A comprehensive history and physical examination, along with other functional tests, are crucial for the evaluation of patients with anterior knee joint pain. ${ }^{21}$ Ethnicity and sex should always be taken into account. In addition, values of the Insall-Salvati ratio for specific ethnic groups are of great importance for accurately evaluating the patellar position.

Our study had several limitations. The first drawback might be sampling bias. Recruitment of patients undergoing MRIs for symptomatic knees may bias the sample. Sampling in the normal population would be ideal. However, that is impossible, both technically and ethically. The second limitation is the difference in the numbers of men and women and the ages of the two sexes. The reason for this is that young men are more often involved in intense physical activities; thus, men are more prone to knee pain and/or injury at an earlier age than women, who usually experience knee pain due to degenerative arthritis. The third limitation is the constricted sample size in a single-institution study. A multi-institutional survey would be more broadly representative of the population as a whole.

\section{Conclusion}

The Insall-Salvati ratio is applicable to the Vietnamese population, in which high flexion activities such as squatting, kneeling, and sitting cross-legged are customary. According to our data, the normal range of the Insall-Salvati ratio among the Vietnamese population was 0.72 to 1.32 . The frequencies of patella alta and patella baja were $0.9 \%$ and $2.4 \%$, respectively.

\section{Abbreviations}

MRI, magnetic resonance imaging; LL, patellar ligament length; PL, patellar length; SD, standard deviation.

\section{Acknowledgments}

This work was supported by Hue University under the Core Research Program, Grant No. NCM.DHH.2020.09.

\section{Disclosure}

The authors report no conflicts of interest in this work.

\section{References}

1. Sanchis-Alfonso V, McConnell J, Monllau JC, Fulkerson JP. Diagnosis and treatment of anterior knee pain. J ISAKOS. 2016;1 (3):161-173. doi:10.1136/jisakos-2015-000033

2. Slotkin S, Thome A, Ricketts C, Georgiadis A, Cruz AI, Seeley M. Anterior knee pain in children and adolescents: overview and management. J Knee Surg. 2018;31(5):392-398. doi:10.1055/ s-0038-1632376

3. Sanchis-Alfonso V, Dye SF. How to deal with anterior knee pain in the active young patient. Sports Health. 2017;9(4):346-351. doi: $10.1177 / 1941738116681269$

4. Werner S. Anterior knee pain: an update of physical therapy. Knee Surg Sports Traumatol Arthrosc. 2014;22(10):2286-2294. doi:10.1007/s00167-014-3150-y

5. Fox AJS, Wanivenhaus F, Rodeo SA. The basic science of the patella: structure, composition, and function. J Knee Surg. 2012;25 (2):127-141. doi:10.1055/s-0032-1313741

6. Insall J, Salvati E. Patella position in the normal knee joint. Radiology. 1971;101(1):101-104. doi:10.1148/101.1.101

7. Shabshin N, Schweitzer ME, Morrison WB, Parker L. MRI criteria for patella alta and baja. Skeletal Radiol. 2004;33(8):445-450. doi:10.1007/s00256-004-0794-6

8. Miller TT, Staron RB, Feldman F. Patellar height on sagittal MR imaging of the knee. AJR Am J Roentgenol. 1996;167(2):339-341. doi:10.2214/ajr.167.2.8686598

9. Hong H-T, Koh Y-G, Nam J-H, Kim PS, Kwak YH, Kang K-T. Gender differences in patellar positions among the Korean population. 2020;10(24):8842.

10. Upadhyay S, Raza HKT, Srivastava P. Position of the patella in adults in central India: evaluation of the Insall-Salvati ratio. J Orthop Surg (Hong Kong). 2013;21(1):23-27. doi:10.1177/230949901302100108

11. Althani S, Shahi A, Tan T, Al-Belooshi A. Position of the Patella among Emirati Adult Knees. Is Insall-Salvati ratio applicable to middle-easterners? Archives Bone Joint Surg. 2016;4(2):137-140. doi:10.22038/abjs.2016.5477

12. Apostolopoulos AP, Najim WS, Fanous R, et al. Patella height in different ethnic populations: an observational multicenter study. J Long Term Eff Med Implants. 2019;29(3):169-173. doi:10.1615/ JLongTermEffMedImplants.2019032873

13. Olateju OI, Philander I, Bidmos MA. Morphometric analysis of the patella and patellar ligament of South Africans of European ancestry. S Afr J Sci. 2013;109(9-10):01-06. doi:10.1590/sajs.2013/20130069 
14. Schlenzka D, Schwesinger G. The height of the patella: an anatomical study. Eur J Radiol. 1990;11(1):19-21. doi:10.1016/0720-048x (90)90097-u

15. Ahmed AD. Radiological assessment of the patella position in the normal knee joint of adult Nigerians. West Afr J Med. 1992;11 (1):29-33.

16. Ra Y, Ea A, Ma T. Patellar height measurements on radiograph and magnetic resonance imaging in patellar instability and control patients. J Knee Surg. 2017;30:930-950. doi:10.1055/s-0037-1599249

17. Neogi DS, Bae JH, Seok CW, Lim HC. Impact of patellar height on unicompartment knee arthroplasty: does patella baja lead to an inferior outcome? J Orthopaed Traumatol. 2014;15(1):47-54. doi:10.1007/ s10195-013-0268-5

18. Ward SR, Powers CM. The influence of patella alta on patellofemoral joint stress during normal and fast walking. Clin Biomech (Bristol, Avon). 2004;19(10):1040-1047. doi:10.1016/j.clinbiomech.2004.07.009
19. Otsuki S, Okamoto Y, Murakami T, et al. Patellofemoral reconstruction for patellar instability with patella alta in middle-aged patients: clinical outcomes. Orthopaedics Traumatol. 2018;104(2):217-221. doi:10.1016/j.otsr.2018.01.003

20. Sanchis-Alfonso V, Coloma-Saiz J, Herrero-Herrero M, PradesPiñón J, Ramírez-Fuentes C. Evaluation of anterior knee pain patient: clinical and radiological assessment including psychological factors. Ann Joint. 2018;3(3). doi:10.21037/aoj.2018. 03.15

21. McCarthy MM, Strickland SM. Patellofemoral pain: an update on diagnostic and treatment options. Curr Rev Musculoskelet Med. 2013;6(2):188-194. doi:10.1007/s12178-013-9159-x

\section{Publish your work in this journal}

Orthopedic Research and Reviews is an international, peer-reviewed, open access journal that focusing on the patho-physiology of the musculoskeletal system, trauma, surgery and other corrective interventions to restore mobility and function. Advances in new technologies, materials, techniques and pharmacological agents are particularly welcome. The manuscript management system is completely online and includes a very quick and fair peer-review system, which is all easy to use. Visit http://www.dovepress.com/testimonials.php to read real quotes from published authors. 